\title{
Effect of N'-Acetylindirubin on Proliferation, Apoptosis and Cell Cycle in Acute Myeloid Leukemia HL-60 Cells
}

\author{
Tang Li1, Li Yu ${ }^{2 *}$ \\ ${ }^{1}$ Department of Pathophysiology, Chongqing Medical University, Chongqing, China \\ ${ }^{2}$ Department of Pathology, Institute of Neuroscience, Chongqing Medical University, Chongqing, China \\ Email: tanglicq@126.com, *liyu100@163.com
}

How to cite this paper: $\mathrm{Li}, \mathrm{T}$. and $\mathrm{Yu}, \mathrm{L}$. (2018) Effect of N'-Acetylindirubin on Proliferation, Apoptosis and Cell Cycle in Acute Myeloid Leukemia HL-60 Cells. Journal of Biosciences and Medicines, 6, 136-141.

https://doi.org/10.4236/jbm.2018.65015

Received: April 24, 2018

Accepted: May 28, 2018

Published: May 31, 2018

\begin{abstract}
Acute promyelocytic leukemia (APL) is a severe type of acute leukemia and the prognosis of patients was poor. Indirubin is the active constituent of the traditional Chinese medicine qingdai and an indoline anti-tumor drug. $\mathrm{N}$ '-Acetylindirubin is a novel indirubin derivative with better curative effect and less side effect. In this study, the effects of N'-Acetylindirubin on proliferation, apoptosis and cell cycle of acute myeloid leukemia cell line HL-60 was examined. The results demonstrated that $\mathrm{N}^{\prime}$-Acetylindirubin significantly induced apoptotic cell death in a dose and time-dependent manner and arrested cell cycle in G2/M in HL-60 cells. N'-Acetylindirubin also suppressed cyclin D1. This study suggests that N'-Acetylindirubin may serves as a potential chemopreventive agent for acute promyelocytic leukemia.
\end{abstract}

\section{Keywords}

N'-Acetylindirubin, HL-60, Apoptosis, Cell Cycle

\section{Introduction}

Acute promyelocytic leukemia (APL) is a severe type of acute leukemia and the treatment is mainly chemotherapy and radiotherapy. The drugs commonly used in chemotherapy have many side effects and the prognosis of patients was still poor. The search for new anticancer compounds in foods or plant medicines is a realistic and promising approach to the prevention and treatment of cancer. Natural products have been considered as valuable sources for anticancer drug discovery [1]. Indirubin is the active constituent of the traditional Chinese medicine qingdai and an indoline anti-tumor drug, mainly used in the treatment of 
chronic myelogenous leukemia [2]. The side effects are mild abdominal pain, abdominal distension, diarrhea, nausea, vomiting and hematoplegia in some patients. There are also severe bone marrow suppression, increased transaminase and headache in patients. N'-Acetylindirubin is a novel indirubin derivative. The pharmacological tests showed significant inhibitory effects on rats and mice tumor. The clinical trial was used to treat chronic myeloid leukemia, and its curative effect was better than indirubin and the side effect was less. In this study, HL-60 cells were used to observe the anti-tumor effect and its mechanism of N'-Acetylindirubin.

\section{Materials and methods}

\subsection{Chemicals}

$\mathrm{N}$-Acetylindirubin was a generous gift from Chongqing Institute of Chinese Materia Medica. Dimethyl sulfoxide (DMSO) and MTT were purchased from Sigma-Aldrich (Bangalore, India). RPMI 1640, fetal bovine serum (FBS) and penicillin-streptomycin solution were purchased from HyClone Laboratories (South Logan, UT, USA).

\subsection{Cell Culture}

The acute myeloblastic leukemia cell line HL-60 was obtained from the American Type Culture Collection (Rockville, MD, USA). Cells were cultured in RPMI 1640 containing $10 \% \mathrm{FBS}, 2 \mathrm{mM}$ glutamine, $100 \mu \mathrm{g} / \mathrm{ml}$ streptomycin, and 100 $\mathrm{U} / \mathrm{ml}$ penicillin.

\subsection{Cell Proliferation Assay}

The effect of N'-Acetylindirubin on cell proliferation was assessed by MTT assay. HL-60 cells were seeded in triplicate in 96-well plates at a density of $1 \times 10^{4}$ cells per well. N'-Acetylindirubin was dissolved in DMSO and diluted in RPMI 1640. The cells were treated with $10,20,30,40$ and $50 \mu \mathrm{M}$ of N'-Acetylindirubin for 24,48 and $72 \mathrm{~h}$. After indicated times, $10 \mu \mathrm{l}$ of MTT solution was added to each well and incubated for $4 \mathrm{~h}$ at $37^{\circ} \mathrm{C} .20 .0 \mu \mathrm{l}$ of DMSO was added to each well. The absorbance was quantified using enzyme-linked immunosorbent assay (ELISA) reader at $570 \mathrm{~nm}$.

\subsection{Flow Cytometric Analysis}

HL-60 cells at $5 \times 10^{5}$ cells $/ \mathrm{ml}$ were inoculated into 6-well culture plate and incubated at $37^{\circ} \mathrm{C}$. The next day, after the medium was removed, $2 \mathrm{ml}$ of RPMI 1640 complete medium with $40 \mu \mathrm{M} \mathrm{N}$ '-Acetylindirubin was added to each well. After cultured for 24,48 , and $72 \mathrm{~h}$, cells were harvested by trypsinization, washed three times with PBS, and suspended in $500 \mu \mathrm{l}$ binding buffer. PI (50 $\mathrm{mg} / \mathrm{ml}, 5 \mathrm{ml}$ ) was added and followed by incubation at room temperature in dark for $30 \mathrm{~min}$. The apoptosis rate was immediately measured by FACSCalibur (BD Biosciences, USA). To evaluate the effect of N'-Acetylindirubin on the cell 
cycle, the harvested cells were washed twice with ice-cold PBS, fixed with ice-cold $70 \%$ ethanol and maintained overnight at $-20^{\circ} \mathrm{C}$. DNA was stained with $100 \mu \mathrm{g} / \mathrm{ml}$ propidium iodide (PI) solution. The cell cycle distribution was analyzed by flow cytometry.

\subsection{Western Blot Analysis}

HL-60 cells were treated with $40 \mu \mathrm{M}$ N'-Acetylindirubin for $48 \mathrm{~h}$. The cells were washed with PBS and then lyzed in RIPA buffer $(150 \mathrm{mM}$ of $\mathrm{NaCl}, 1 \mathrm{mM}$ of EDTA, $50 \mathrm{mM}$ of Tris- $\mathrm{HCl}$ at $\mathrm{pH} 7.4,0.5 \%$ sodium deoxycolate, $1 \%$ Nonidet $\mathrm{P}-40,0.1 \%$ SDS), containing protease inhibitors. Lysates were ultracentrifuged at $14,000 \mathrm{~g}$ for $15 \mathrm{~min}$ at $4^{\circ} \mathrm{C}$, the supernatants were collected as the cell extracts. Protein concentration was determined by BCA protein assay and lysates were subjected to $8 \%-12 \%$ SDS-PAGE and transferred onto a PVDF membrane. After blocking with $5 \%$ non-fat milk in tris-buffered saline with $0.1 \%$ Tween 20 (TBST), membranes were incubated with respective primary antibodies directed against CyclinD1 (1:1000) and $\beta$-actin $(1: 3000)$ at $4^{\circ} \mathrm{C}$ overnight. Membranes were washed and then incubated with HRP-conjugated rabbit anti-IgG (1:5000) for $1 \mathrm{~h}$ at room temperature. Protein bands were assessed by enhanced chemiluminescence system (ECL, KeyGEN, China).

\subsection{Statistical Analysis}

All the data were expressed as means \pm standard deviation (SD). All statistical analyses were evaluated using SPSS 17.0 software. The significance of difference between the groups was analyzed with two-way ANOVA test or two-tailed unpaired Student's t-test. P-values $<0.05$ was considered as statistically significant.

\section{Results}

\subsection{N'-Acetylindirubin Inhibits Cell Proliferation}

The effect of N'-Acetylindirubin on HL-60 cells was investigated by MTT assay. The results showed that $\mathrm{N}^{\prime}$-Acetylindirubin inhibited the proliferation of HL-60 cells in dose- and time-dependent manners (Table 1). The cytostatic does of 40 $\mu \mathrm{M}$ for $48 \mathrm{~h}$ was taken for further study of N'-Acetylindirubin on HL-60 cells.

\subsection{N'-Acetylindirubin Induces Cell Apoptosis}

Apoptosis was also detected by flow cytometric analysis using the PI staining. As shown in Table 2, $\mathrm{N}$ '-Acetylindirubin $40 \mu \mathrm{M}$ promoted HL-60 cells apoptosis after an exposure of both 48 and 72 hours (16.3\% and $23.2 \%$ of cells, respectively).

\subsection{N'-Acetylindirubin Arrests Cell Cycle}

To further study the effect of $\mathrm{N}$ '-Acetylindirubin on the cell cycle, HL-60 cells were analyzed by flow cytometry after 12,24 and $48 \mathrm{~h}$ of treatment with $40 \mu \mathrm{M}$ $\mathrm{N}^{\prime}$-Acetylindirubin. It showed that $16.84 \%, 25.51 \%$ and $30.37 \%$ of cells were 
Table 1. Inhibitory Effect of N'-Acetylindirubin on the growth of HL-60 cell.

\begin{tabular}{cccc}
\hline & $24 \mathrm{~h}$ & $28 \mathrm{~h}$ & $72 \mathrm{~h}$ \\
\hline $10 \mu \mathrm{M}$ & $0.20 \pm 0.08$ & $0.15 \pm 0.05$ & $0 \pm 0.00$ \\
$20 \mu \mathrm{M}$ & $0.25 \pm 0.02$ & $0.06 \pm 0.02$ & $0.32 \pm 0.05$ \\
$30 \mu \mathrm{M}$ & $0.30 \pm 0.09$ & $0.21 \pm 0.01$ & $0.51 \pm 0.06$ \\
$40 \mu \mathrm{M}$ & $0.62 \pm 0.01$ & $0.55 \pm 0.00$ & $0.82 \pm 0.02$ \\
$50 \mu \mathrm{M}$ & $0.68 \pm 0.03$ & $0.62 \pm 0.00$ & $0.91 \pm 0.00$ \\
\hline
\end{tabular}

Table 2. Effect of N'-Acetylindirubin on apoptotic rate of HL-60 cells (\%).

\begin{tabular}{ccc}
\hline & 0 & $40 \mu \mathrm{M}$ \\
\hline $24 \mathrm{~h}$ & $0.7 \pm 0.1$ & $7.7 \pm 1.1$ \\
$48 \mathrm{~h}$ & $1.3 \pm 0.2$ & $16.3 \pm 1.3$ \\
$72 \mathrm{~h}$ & $1.9 \pm 0.7$ & $23.2 \pm 0.9$ \\
\hline
\end{tabular}

reached in G2/M phase comparing to $8.13 \%$ (control). There was a time-dependent increase of cells at the G2/M phase. This indicated that N'-Acetylindirubin induced cell cycle arrest at G2/M phase, which led to proliferation inhibition.

\subsection{N'-Acetylindirubin Decreases Expression of Cyclin D1}

The protein level of cyclin D1 was determined by immunoblotting. As shown in Figure 1, expression of cyclin D1 decreased in HL-60 cell after treated with 40 $\mu \mathrm{M} \mathrm{N}^{\prime}$-Acetylindirubin for $48 \mathrm{~h}$. The change of cyclin D1 was associated with $\mathrm{G} 2 / \mathrm{M}$ phase arrest of the cell cycle. $\beta$-actin served as an internal control.

\section{Discussion}

Indirubin is the active ingredient of qingdai in traditional chinese medicine Danggui Longhui Wan, which has potential anti-tumor activity. Indirubin was started to be used in treatment of chronic myelocytic leukemia (CML). More than half of the treated CML patients exhibited partial or complete remission [3] [4] [5]. The side effects of indirubin were abdominal pain, diarrhea, and nausea [6]. However, poor solubility and low bioavailability have limited its application in clinic. In order to reduce toxic side effects, improve pharmacokinetic properties and increase efficacy, some new indirubin derivatives were synthesized and be studied in other types of cancer as well as other diseases [7] [8] [9]. Here, we investigated the anti-tumor effect and its mechanism of $\mathrm{N}^{\prime}$-Acetylindirubin, a novel indirubin derivative, in acute myeloid leukemia cell line HL-60.

In our present study, we examined the anticancer effect of N'-Acetylindirubin. The result of the cell proliferation assay showed that N'-Acetylindirubin exerts a potent cytotoxic effect on HL-60 in a dose and time-dependent manner. The inhibition of proliferation in HL-60 cells was a result of apoptosis induction and 


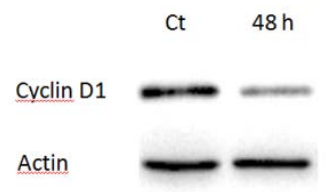

Figure 1. Expression of cell cycle-controlling protein Cyclin D1 in HL-60 cell.

cell cycle arrest. Apoptosis is an important homeostatic mechanism that is characterized by unique morphological and biochemical features and is used maintain the appropriate numbers of cells in the body. Activation of a group of cysteine proteases named "caspases" that play a vital role in the initiation of the death signals leading to apoptosis. Caspases can be divided into initiators, including caspase-1, $-2,-8,-9$ and -10 , which are involved in early stages of the proteolytic cascade, and effector caspases, including caspase-3, -6 and -7 , which are involved in the cleavage of specific intracellular substrates (e.g., poly-ADP-ribose polymerase, focal adhesion kinase). Studies have also suggested induction of expression of caspase 3 is a crucial step in curcumin- and gypenoside-induced apoptosis in colorectal cancer (CRC) cells [10] [11]. LCSP treatment increased the protein level of the active form of caspase 3 in CRC cells, indicating that LCSP-induced apoptosis is mediated by caspase 3 activation. Induction of apoptosis is also one possible mechanism of antiproliferative activity in HL-60 cells. In current study, we demonstrated that $\mathrm{N}^{\prime}$-Acetylindirubin treated cells express an apoptotic reaction. Our investigation of the cell cycle distribution revealed that the cell cycle was arrested in the G2/M phase. The cell cycle is controlled by a group of regulatory proteins named cyclins. Cyclin D1 is an important regulator of G1 phase progression in many different cell types including HL-60 cells [12]. In this study, N'-Acetylindirubin treatment decreased the level of cyclin D1 in HL-60 cells, which is correlated with the cell cycle analysis showing $\mathrm{G} 2 / \mathrm{M}$ phase arrest.

In conclusion, our study demonstrated that $\mathrm{N}^{\prime}$-Acetylindirubin treatment inhibited cell proliferation in HL-60. N'-Acetylindirubin inhibited HL-60 cells mainly through apoptosis and G2/M phase arrest, suggesting its potential as a novel chemoprevention agent for acute promyelocytic leukemia in the future.

\section{References}

[1] Schwartsmann, G., Ratain, M.J., Cragg, G.M., et al. (2002) Anticancer Drug Discovery and Development throughout the World. JClin Oncol, 20, 47S-59S.

[2] Blažević, T., Heiss, E.H., Atanasov, A.G., et al. (2015) Indirubin and Indirubin Derivatives for Counteracting Proliferative Diseases. Evid Based Complement Alternat Med, 2015, Article ID: 654098. https://doi.org/10.1155/2015/654098

[3] Ma, M.Z. and Yao, B.Y. (1983) Progress in Indirubin Treatment of Chronic Myelocytic Leukemia. Journal of Traditional Chinese Medicine, 3, 245-248.

[4] Zhang, Z.N., Liu, E.K., Zheng, T.L., et al. (1985) Treatment of Chronic Myelocytic Leukemia (CML) by Traditional Chinese Medicine and Western Medicine Alternately. Journal of Traditional Chinese Medicine, 5, 246-248. 
[5] Hoessel, R., Leclerc, S., Endicott, J.A., et al. (1999) Indirubin, the Active Constituent of a Chinese Antileukaemia Medicine, Inhibits Cyclin-Dependent Kinases. Nature Cell Biology, 1, 60-67. https://doi.org/10.1038/9035

[6] Xiao, Z., Hao, Y., Liu, B., et al. (2002) Indirubin and Meisoindigo in the Treatment of Chronic Myelogenous Leukemia in China. Leukemia \& Lymphoma, 43, 1763-1768. https://doi.org/10.1080/1042819021000006295

[7] Karapetyan, G., Chakrabarty, K., Hein, M., et al. (2011) Synthesis and Bioactivity of Carbohydrate Derivatives of Indigo, Its Isomers and Heteroanalogues. Chem Med Chem, 6, 25-37. https://doi.org/10.1002/cmdc.201000374

[8] Vougogiannopoulou, K. and Skaltsounis, A.L. (2012) From Tyrian Purple to Kinase Modulators: Naturally Halogenated Indirubins and Synthetic Analogues. Planta Medica, 78, 1515-1528. https://doi.org/10.1055/s-0032-1315261

[9] Wang, Y., Hoi, P.M., Chan, J.Y.W., et al. (2014) New Perspective on the Dual Functions of Indirubins in Cancer Therapy and Neuroprotection. Anti-Cancer Agents in Medicinal Chemistry, 14, 1213-1219.

https://doi.org/10.2174/1871520614666140825112924

[10] Su, C.C., Lin, J.G., Li, T.M., et al. (2006) Curcumin-Induced Apoptosis of Human Colon Cancer Colo 205 Cells through the Production of ROS, $\mathrm{Ca}^{2+}$ and the Activation of Caspase-3. Anticancer Research, 26, 4379-4389.

[11] Chen, J.C., Lu, K.W., Lee, J.H., et al. (2006) Gypenosides Induced Apoptosis in Human Colon Cancer Cells through the Mitochondria-Dependent Pathways and Activation of Caspase-3. Anticancer Research, 26, 4313-4326.

[12] Alao, J.P. (2007) The Regulation of Cyclin D1 Degradation: Roles in Cancer Development and the Potential for Therapeutic Invention. Molecular Cancer, 6, 24. https://doi.org/10.1186/1476-4598-6-24 